\title{
Bacterial Protein Mediated Synthesis of Antibacterial AgNPs and Protein Crystallization for Time-dependent Growth Studies of AgNPs
}

\author{
Noreen Ashraf, Fiaz Ahmad, Yin Da-Chuan \\ Institute for Special Environmental Biophysics, Key Laboratory for Space Bioscience and Biotechnology \\ School of Life Sciences, Northwestern Polytechnical University \\ Xi' an 710072, Shaanxi, PR China \\ noreenashrafnpu@yahoo.com; fiaz.a@mail.nwpu.edu.cn; yindc@nwpu.edu.cn
}

\section{Extended Abstract}

The increasing demand for nanoparticles has attracted researchers to develop straightforward, inexpensive, simple, and eco-friendly processes for their synthesis. Based on distinct optical properties and chemical stability silver nanoparticles (AgNPs) are useful in diverse biomedical applications. Mainly, AgNPs are capable of influencing microbial growth and show broad-spectrum antimicrobial potential $[1,2]$. In medical industry, AgNPs are used as topical ointments in open wounds and burns to prevent infections. However, the knowledge of nucleation, structure of AgNPs and proteins acting as a scaffold for the synthesis of AgNPs is a key to understanding the in-depth mechanism of a biological process such as mode of action of AgNPs and development of therapeutics, etc. One of the methods used to determine structures of proteins and nanocrystals of AgNPs is X-ray crystallography [3,4]. Studies regarding biological synthesis of AgNPs from silver salts has also led to an increased level of interest in the bio-mineralization of silver. While the underlying mechanisms responsible for the biomolecule-directed synthesis of AgNPs remains unclear due to the lack of structural information of involved biomolecules and the fast kinetics of biogenic chemical systems in solutions.

The objective of this study was the synthesis and characterization of biogenic AgNPs by a novel bacteria strain Enterobacter cloacae (SMP1) isolated from the silver mining site. The antibacterial assay was performed using welldiffusion method against human pathogens including Enterobacter cloacae, Pseudomonas aeruginosa, Escherichia coli, Klebsiella pheumoniae, and Staphylococcus aureus, which were resistant to various commercially available antibiotics. Also, we optimized experimental conditions to enhance the expression of native extracellular proteins of SMP1 strain to grow protein crystals directly from impure protein sample to use them for time-dependent growth of AgNPs within single crystals of protein.

Synthesized AgNPs were purified by centrifugation at 22,000 $\mathrm{g}$ with subsequent washings and analyzed through UVvisible spectrophotometry, dynamic light scattering (DLS), atomic force microscopy (AFM), SEM and TEM. Also, Extracellular proteins responsible for the synthesis of AgNPs determined by SDS-PAGE, and a protein with higher expression with a size of $16 \mathrm{kDa}$ was identified through LC-MS analysis. Protein crystallization screening trials were carried out by using commercially available protein crystallization kits, and protein crystallization conditions were selected and optimized for better crystal growth.

Results showed that the biogenic AgNPs were crystalline ranging in size from 10-70 nm. Importantly, antibacterial assay exhibited concentration-dependent bactericidal effect of AgNPs on all tested human pathogenic strains, while all of these were resistant against tested antibiotics. LC-MS analysis of protein with maximum expression identified it as inosine monophosphate dehydrogenase (ImpD) related protein (a novel protein that has not been characterized yet). Moreover, we were lucky enough to grow protein crystals, which will allow us to perform time-dependent growth of AgNPs within native protein crystals.

This study revealed that the biogenic AgNPs synthesized by Enterobacter sp. are the potential candidates for their use in the field of medicine. Crystallization of native proteins showed strong interaction with silver metal ions may open up a new horizon for detailed investigation of nucleation of AgNPs and helps in understanding the mechanism of protein-metal interactions. Also, elucidation of protein structure will be a significant achievement in the field of protein crystallization and structural biology. 


\section{References}

[1] M. Rai, A. Yadav, A. Gade, "Silver nanoparticles as a new generation of antimicrobials," Biotechnol. Adv., vol. 27, pp. 76-83, 2009.

[2] B. Momin, S. Rahman, N. Jha, U. S. Annapure, "Valorization of mutant Bacillus licheniformis M09 supernatant for green synthesis of silver nanoparticles: photocatalytic dye degradation, antibacterial activity, and cytotoxicity," Bioprocess Biosyst. Eng., pp. 1-13, 2019.

[3] C. Wang, Y. J. Kim, P. Singh, R. Mathiyalagan, Y. Jin, D. C. Yang, "Green synthesis of silver nanoparticles by Bacillus methylotrophicus, and their antimicrobial activity, Artif. Cells Nanomed," Biotechnol., vol. 44, pp. 11271132, 2016.

[4] G. Ferraro, T. Marzo, M. E. Cucciolito, F. Ruffo, L. Messori, A. Merlino, "Reaction with proteins of a five-coordinate platinum(II) compound," Int. J. Mol. Sci., vol. 20, no. 3, pp. 520, 2019. 